\title{
The Electrostatic Nature of Gravitation
}

\author{
V. K. Gudym ${ }^{1} \&$ E. V. Andreeva ${ }^{2}$ \\ ${ }^{1}$ National Space Agency of Ukraine, Kyiv, Ukraine \\ ${ }^{2}$ Institute of Semiconductor Physics of the NASU, Kyiv, Ukraine \\ Correspondence: V. K. Gudym, National Space Agency of Ukraine, Kyiv 01010, Ukraine. E-mail: \\ vgudym@mail.ru
}

Received: August 5, 2013 Accepted: September 30, 2013 Online Published: November 5, 2013

doi:10.5539/apr.v5n6p93 URL: http://dx.doi.org/10.5539/apr.v5n6p93

\begin{abstract}
In work on the basis of static model of a structure of atoms it is shown that the gravitational and electrostatic interactions have the same nature. The electrostatic interaction is the interaction of electric charges at small distances, whereas the gravitational interaction is the interaction of electric charges at large distances.
\end{abstract}

Keywords: the gravitation nature, a binomial potential, static model of atom, the scattering of electrons by protons

\section{Introduction}

At the present time, the gravity remains the most puzzling phenomenon of the Nature. The paradox consists in that the gravitation involves all subjects existing in the world, from the Universe itself and to its microcomponents. However, no knowledge of the physical essence of the gravitation and a relation to other known interactions is available. There exist a lot of opinions on this question. We present one of the most characteristic thoughts. The answer of Carver Mead to the question: "Do you have any thoughts about gravitation?" was “... we basically have no clue what it is. It doesn't fit with any of the other theories" (Mead, 2001).

We believe that the overcoming of difficulties in the comprehension of the gravitational interaction requires to reject completely the planetary model of the structure of atoms and molecules. Instead of the planetary model, we propose a model based on the binomial potential of the electron-proton interaction (Gudym \& Andreeva, 2007, 2008). In essence, we propose a static model of an atom, in which an electron and a proton and, in the general case, the nucleus and atomic electrons, undergo the action of two oppositely directed forces. One of the forces is responsible for the attraction of electric charges, and another one repulses them. These forces are equal in modulus at some distance from the nucleus which can be called the size of an atom. Moreover, the forces of repulsion in our model with the binomial potential are short-range forces. Therefore, the interaction between an atom as a whole and remote charges outside of the atom is mainly attractive, because the short-range repulsive forces at large distances are significantly less than the attractive ones. Hence, the electrically neutral atom, molecule, or any macro body, while interacting with electric charges of the other analogous systems, undergo only the electrostatic attraction at distances much more than the size of an atom. Just this is the essence of the proposed mechanism of gravitational interaction.

Since the physical basis of the appearance of the gravitational interaction is given by the binomial potential of the interaction of an electron and a proton, our main task was to demonstrate the reality of the binomial potential by specific examples. To this end, we considered the classical problem on the motion of an electron in the field of the binomial potential of a proton and solved the Schrödinger equation with this potential (Gudym \& Andreeva, 2008). Then we calculated the energies of ten first atoms of the Mendeleev Periodic table within the model of the binomial potential (Gudym \& Andreeva, 2008). The results of calculations supported our confidence in the reality of the binomial potential.

Then, on the basis of models of electrically neutral atoms (Gudym \& Andreeva, 2008), we developed a model of interaction of these atoms and free charges. The study of near-atomic regions within those models allowed us to surely conclude that any electrically neutral atom and any electric charge positioned from it at any distance attract each other. 
Hence, the forces of attraction acting between physical bodies (gravity force) are a result of the electrostatic interaction of electrically neutral atoms with electric charges of other, also electrically neutral, atoms.

\section{Substantiation of the Binomial Potential}

As a basis of the binomial potential of the interaction of an electron and a proton, we took the indisputable fact that the electrons in an atom are located at some distances from the nucleus. In this case, the interaction potential for the electron and the proton in a hydrogen atom can be presented by the function

$$
V=-\frac{e^{2}}{r}+\frac{\Gamma}{r^{x}}
$$

where the first and second terms on the right-hand side represent, respectively, the Coulomb interaction and our hypothetical interaction which counteracts the Coulomb attraction.

To determine the constant $\Gamma$ and the exponent $x$, we constructed a system of two algebraic equations corresponding to the equilibrium (ground) state of a hydrogen atom according to the scheme in (Born, 1946):

$$
-\frac{e^{2}}{r_{0}}+\frac{\Gamma}{r_{0}^{x}}=E_{0}, \frac{e^{2}}{r_{0}^{2}}-\frac{x \cdot \Gamma}{r_{0}^{x+1}}=0
$$

Here, $E_{0}$ is the ground state energy of a hydrogen atom, $r_{0}$ is its equilibrium radius, and $e$ is the electron charge. The second relation in (2) is the sum of the forces acting on the electron in the ground state.

The solution (Appendix A) of system (2) for $\Gamma$ and $x$ gave the following results:

$$
\Gamma=6.10276 \cdot 10^{-28} \text { CGSE units and } x=2
$$

Generally speaking, within the model of binomial potential, we replace, in fact, the postulate about some motion of an electron around the nucleus, which is extraneous to classical mechanics, by a more natural hypothesis about the presence of a repulsive potential. Moreover, a particular attention should be given to the circumstance that the proposed binomial potential (1) does not contradict the foundations of quantum mechanics (Gudym \& Andreeva, 2008) and open a way of classical mechanics to many-electron systems.

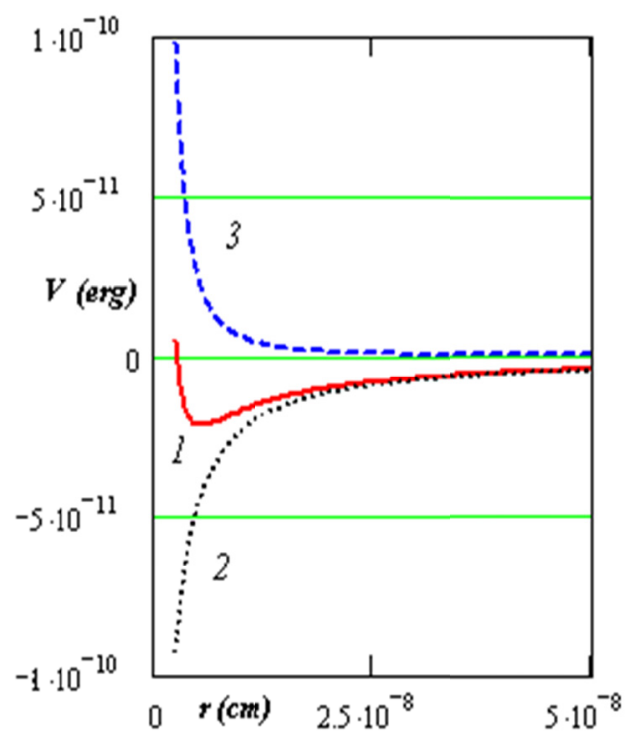

Figure 1. Interaction energy of an electron and a proton the distance between them (1), the Coulomb component (2), the hypothetical component (3)

In Figure 1, we present the dependence of the interaction energy of an electron and a proton in the binomial-potential model on the distance between them. It is obvious that the Coulomb component dominates in the total interaction energy already at distances of the order of magnitude of two equilibrium radii $\left(\sim 1 \times 10^{-8} \mathrm{~cm}\right)$. But at small distances, approximately at half of the equilibrium radius $\left(\sim 0.25 \times 10^{-8} \mathrm{~cm}\right)$, the repulsive component becomes dominant. Hence, the positive addition to the Coulomb term in (1) represents a short-range 
force. Apparently, just in this way it is possible to explain the fact that no deviations from the Coulomb law were found at macro distances.

\section{The Two-Body Problem with Binomial Potential}

On the next stage of our study, it was necessary to verify the reality of the binomial potential (1). With this purpose, we considered the two-body problem with the binomial potential and solved the equation (Gudym \& Andreeva, 2007).

$$
E=\frac{m \dot{r}^{2}}{2}+\frac{M^{2}}{2 m r^{2}}-\frac{e^{2}}{r}+\frac{\Gamma}{r^{2}},
$$

where $m$ is the electron mass, $E$ is the total energy of the system, $M$ is the angular momentum, and $\dot{r}$ is the derivative with respect to time.

The solution (Appendix B) of Equation (4) for a trajectory has the form

$$
r=\frac{P}{1-\varepsilon \sin (k \phi)},
$$

where

$$
\varepsilon=\sqrt{1+\frac{2 E\left(2 m \Gamma+M^{2}\right)}{m e^{4}}}, \quad P=\frac{2 m \Gamma+M^{2}}{m e^{2}}, k=\frac{\sqrt{2 m \Gamma+M^{2}}}{M} .
$$

In the general case, Function (5) represents two types of motion, finite and infinite ones, by depending on the sign of the energy in Equation (4). The former is the motion of a bound electron in a hydrogen atom, and the latter corresponds to the scattering of an electron by a proton.

\subsection{Hydrogen Atom}

Let us consider firstly a finite motion. The solution of (4) differs from the well-known solution of the Kepler problem with the Coulomb potential only by the presence of the coefficient $k$ of the argument $\varphi$. In the general case, the presence of this coefficient leads to the appearance of a circular motion of the perihelion. It follows from relations (6) that if the positive term in (1) is dropped, then $k=1$, and the trajectory corresponds to a conical cross-section with the focus at the origin of coordinates. The nonzero positive term makes a value of the coefficient $k$ to be different from 1 . This means that the trajectory of motion will be unclosed in the general case. Only separate values of the coefficient $k$ will satisfy the conditions of closedness. In other words, if the electron with any energy in the Coulomb field can move only along a closed orbit, its motion along a closed orbit in potential (1) can be realized only at strictly definite values of the energy (Born,1946).

By solving Equation (4) under the condition $E<0$, we succeeded to obtain the discreteness of energy levels, Balmer formula, and Planck's constant and relation (Gudym \& Andreeva, 2013, 2007).

\subsection{Scattering of Electrons by Protons}

If the total energy (4) is positive, then the motion trajectory of an electron in the central field of a proton will be an unclosed curve, whose beginning and end are at infinity. Since the motion is infinite in this case, it is convenient to introduce the so-called impact parameter $\rho$ instead of the constant angular momentum $M$ :

$$
M=\sqrt{2 m E \rho^{2}} .
$$

To describe the trajectory of motion of the electron scattered by the proton, we substitute (7) in relations (6) and pass to the Cartesian coordinates

$$
x=r \cdot \cos \varphi, y=r \cdot \sin \varphi
$$

where the values of $r$ should be determined by Formula (5).

In Figure 2, we show the trajectories of motion of scattered electrons with energies of 400, 188 , and $40 \mathrm{MeV}$ calculated by (8). It is seen that if the motion occurs in the Coulomb potential field, no scattering of electrons with such energies is observed. 


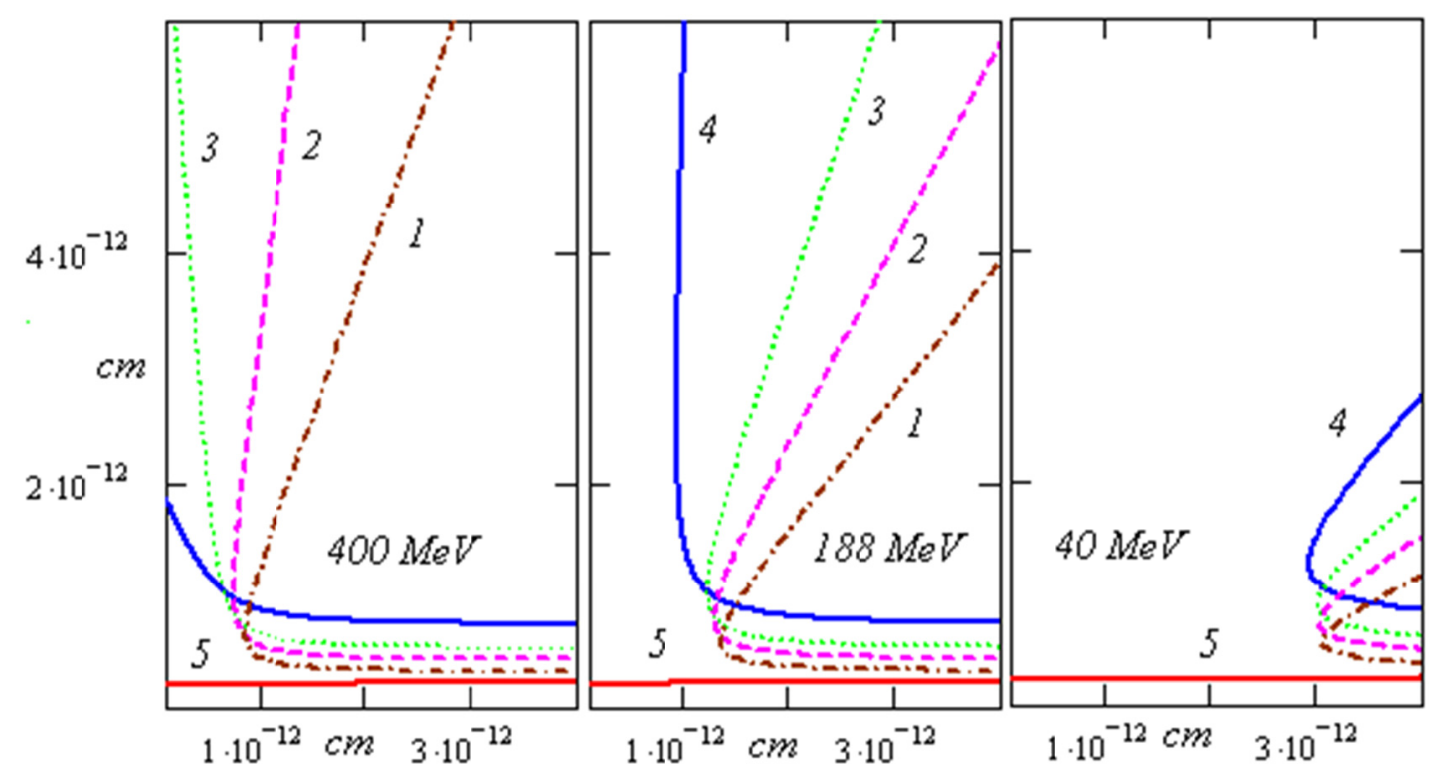

Figure 2. Trajectories of motion of electrons with energies of 400, 188 and $400 \mathrm{MeV}$ scattered by a proton. Calculations are performed by formula (8) for impact parameters $\rho: 1) 4 \times 10^{-13}$;2) $5 \times 10^{-13}$; 3) $6 \times 10^{-13}$; 4) $8 \times$ $\left.10^{-13} \mathrm{~cm} ; 5\right)$ a trajectory of motion of an electron in the Coulomb potential

The deviation angle $\chi$ of a particle moving near the scattering center can be presented as

$$
\chi=\pi-2 \varphi_{0}
$$

where the angle $\varphi_{0}$ is given by the integral

$$
\phi_{0}=\int_{r_{\min }}^{\infty} \frac{M \cdot d r}{r^{2} \sqrt{2 m[E-U(r)]-\frac{M^{2}}{r^{2}}}}
$$

taken between the position of the particle which is the closest to the scattering center and the infinity (Lavich, 1969). The value of $r_{\min }$ is a root of the radicand.

With regard for Relation (7), Formula (10) takes the form

$$
\phi_{0}=\int_{r_{\min }}^{\infty} \frac{d r}{r^{2} \sqrt{\frac{1}{\rho^{2}}\left[1-\frac{U(r)}{E}\right]-\frac{1}{r^{2}}}} .
$$

By substituting potential (1) in Formula (11) and carrying out the integration, we obtain

$$
\varphi_{0 b}=\sqrt{\frac{E \rho^{2}}{E \rho^{2}+\Gamma}} \cdot \arccos \left[\frac{1}{\sqrt{1+\frac{4 E\left(E \rho^{2}+\Gamma\right)}{e^{4}}}}\right]
$$

Here, the letter $b$ in the index of the angle $\varphi_{0 b}$, means that the angle is determined within the binomial-potential model (1).

Thus, we obtained the analytic formula for the deviation angle $\chi$ for the electron scattered by the proton as a function of its kinetic energy $E$ and the impact parameter $\rho$ under the assumption that they interact by the law with the binomial potential (1). If we set $\Gamma=0$ in Formula (12), which corresponds to the Coulomb law for of the electron-proton interaction (Levich, 1969) we get 


$$
\varphi_{0 k}=\arccos \left[\frac{1}{\sqrt{1+\frac{4 E^{2} \rho^{2}}{e^{4}}}}\right]
$$

Here, the letter $k$ in the index of the angle $\varphi_{0 k}$, indicates that the deviation angle is determined for the Coulomb scattering.

Hence, the difference of the formulas derived with the help of the Coulomb and binomial potentials consists, basically, only in the presence of the coefficient

$$
k=\sqrt{\frac{E \rho^{2}+\Gamma}{E \rho^{2}}}
$$

in (12).

In the general case, Formulas (12) and (13) together with (8) allow one to calculate the deviation angle $\chi$ for a scattered electron as a function of its kinetic energy and the impact parameter in both cases where the electron interacts with the proton by the binomial law (1) and the Coulomb law.

In the experimental studies of the process of scattering, the so-called effective scattering cross-section $d \sigma$ is used. Since relation (12) establishes the bijective interrelation between the deviation angle $\chi$ and the impact parameter $\rho$, the effective scattering cross-section referred to a solid angle element $d \Theta$ can be presented as (Levich, 1969)

$$
d \sigma=\frac{\rho(\chi)}{\sin (\chi)} \cdot \frac{d \rho}{d \chi} \cdot d \theta
$$

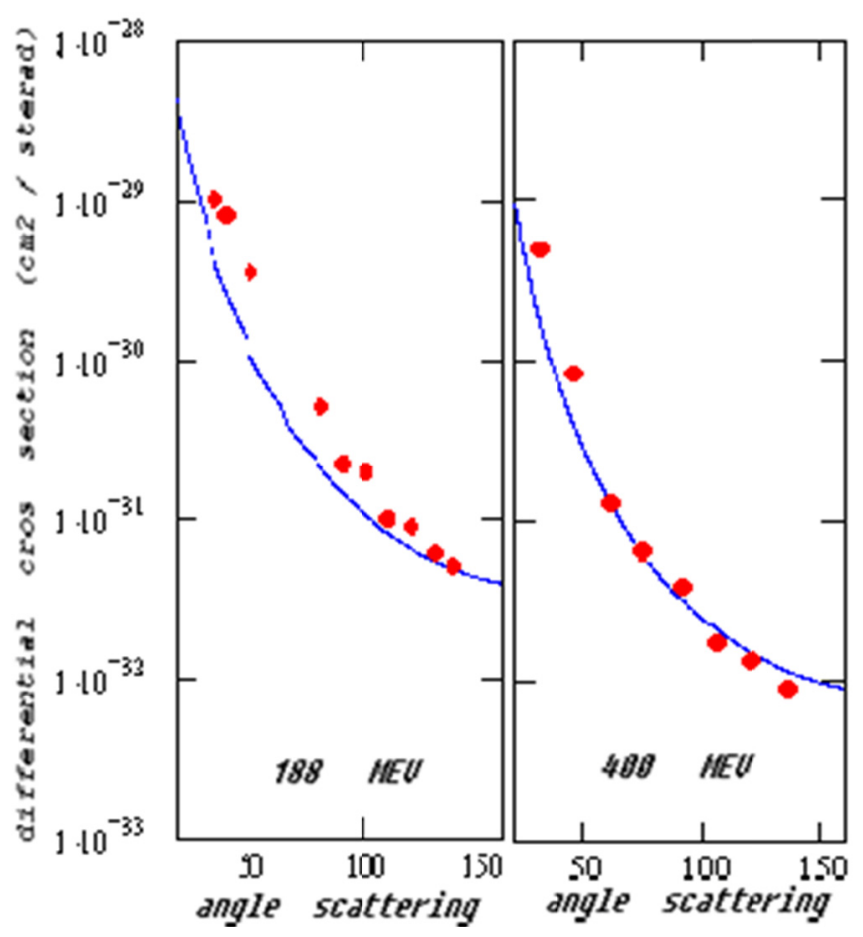

Figure 3. Comparison of the calculated effective cross-sections for electrons with energies of 400 and $188 \mathrm{MeV}$ with experimental values: points - experimen (Hofstadter, 1956); curves - calculated data

Let us introduce the notation $k \phi_{0}=(\pi-\chi) / 2$. The Formula (12) yields

$$
\rho^{2}=\frac{e^{4}}{4 E^{2}} \cdot \operatorname{ctg}^{2}\left(\frac{\chi}{2}\right)-\frac{\Gamma}{E}
$$


By substituting the result of differentiation of Formula (16) in the formula for the effective scattering cross-section (15), we obtain

$$
d \sigma=\frac{e^{4}}{16 E^{2}} \cdot \frac{1}{\sin ^{4}\left(\frac{\chi}{2}\right)} \cdot d \theta
$$

By the form, this formula repeats exactly the Rutherford formula (Levich, 1969). But formula (17) differs basically from the Rutherford one in the definition of the angle $\chi$ with reagard for coefficient (14):

$$
\chi=\pi-2 \kappa \varphi_{0}
$$

In Figure 3, we compare the curve calculated by Formula (17) for the effective scattering cross-section of electrons with energies of 400 and $188 \mathrm{MeV}$ with the experimental data from work (Hofstadter, 1956).

Generally speaking, it is the unexpected result. Indeed, it is commonly accepted (Hofstadter, 1956; Levich, 1969) that the Rutherford formula must not work in the region of relativistic energies. However, the whole sequence of above-performed mathematical operations corresponds exactly to the derivation of the Rutherford formula for the Coulomb potential and indicates that the uses of the binomial and Coulomb potentials yield the same formula for the effective scattering cross-section.

The situation becomes different, if we calculate the trajectories of motion of an electron. It follows from Figure 2 that the results of calculations of the trajectories of motion of high-energy electrons in the Coulomb field are unphysical. On the contrary, the trajectories of motion of electrons with any energy within the binomial-potential model are quite real.

Thus, we manage to deduce a single formula similar to the well-known Rutherford one for the calculation of the trajectories of motion and the scattering angles of electrons by protons for the entire range of energies of the scattered electrons from several $\mathrm{eV}$ up to ultra relativistic energies and with impact parameters down to $10^{-13} \mathrm{~cm}$. It is worth separately noting that our formulas do not require any modification within the other theories for different energies (Hofstadter, 1956; Levich, 1969), which is necessary in the Rutherford approach.

Hence, we may conclude that the binomial potential of the interaction of an electron and a proton reflects more exactly the character of forces acting between an electron and a proton, than the Coulomb potential.

\section{Static Models for Ten First Atoms of the Mendeleev Periodic Table}

To support the reality of the binomial potential, we proposed static on it to basis models of ten first atoms of the Mendeleev Periodic table within our model in the form of geometric plane or volumetric structures, where the nucleus is located at the center of the structure, and the electrons are positioned at its vertices (Figure 4, Gudym \& Andreeva, 2008).

To verify the validity of such models, we calculated the electron interaction energy for each of those atoms.

As an example, we present the formulas for some atoms.

Helium atom:

$$
E_{02}=-\frac{4 e^{2}}{r}+\frac{2 \Gamma}{r^{2}}+\frac{e^{2}}{R}+\frac{A}{R^{2}},
$$

where $E_{02}$ is the electron interaction energy; $r$ is the distance of electrons from the nucleus; $R$ is the distance between electrons; and $A$ is the magnetic interaction constant $\left(A=3.107 \times 10^{-28}\right.$, Gudym \& Andreeva, 2008).

Beryllium atom:

$$
E_{04}=-\frac{8 e^{2}}{r_{1}}-\frac{8 e^{2}}{r_{2}}+\frac{2 \Gamma}{r_{1}^{2}}+\frac{2 \Gamma}{r_{2}^{2}}+\frac{4 e^{2}}{R}+\frac{e^{2}}{2 r_{1}}+\frac{e^{2}}{2 r_{2}}+\frac{4 A}{R^{2}}+\frac{A}{\left(2 r_{1}\right)^{2}}+\frac{A}{\left(2 r_{2}\right)^{2}}
$$

where $E_{02}$ is the electron interaction energy; $R$ is the size of a rhomb edge; $r_{1}$ and $r_{2}$ are the distances of oppositely located electrons from the nucleus which are related to the rhomb edge as follows: $r_{1}=R \sin \alpha$ and $r_{2}=$ $R \cos \alpha$; and $\alpha$ is the angle between an edge and the major diagonal.

To verify the validity of such models, we calculated the electron interaction energy for each of those atoms.

As an example, we present the formulas for some atoms. 
Helium atom:

$$
E_{02}=-\frac{4 e^{2}}{r}+\frac{2 \Gamma}{r^{2}}+\frac{e^{2}}{R}+\frac{A}{R^{2}},
$$

where $E_{02}$ is the electron interaction energy; $r$ is the distance of electrons from the nucleus; $R$ is the distance between electrons; and $A$ is the magnetic interaction constant $\left(A=3.107 \times 10^{-28}\right.$, Gudym \& Andreeva, 2008).

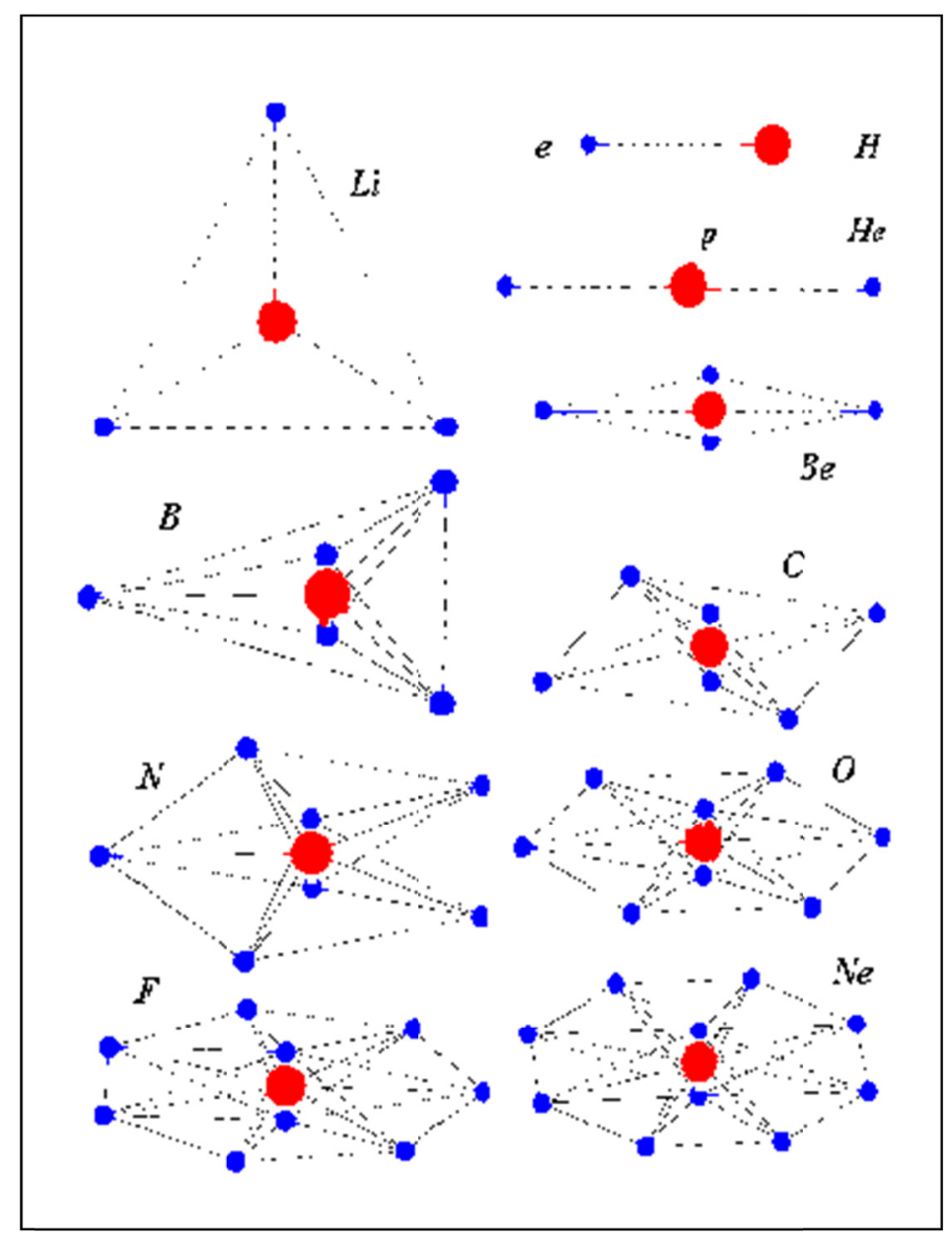

Figure. 4 Static models of atoms; red filled circle — atomic nucleus; blue filled circle_-electron

Beryllium atom:

$$
E_{04}=-\frac{8 e^{2}}{r_{1}}-\frac{8 e^{2}}{r_{2}}+\frac{2 \Gamma}{r_{1}^{2}}+\frac{2 \Gamma}{r_{2}^{2}}+\frac{4 e^{2}}{R}+\frac{e^{2}}{2 r_{1}}+\frac{e^{2}}{2 r_{2}}+\frac{4 A}{R^{2}}+\frac{A}{\left(2 r_{1}\right)^{2}}+\frac{A}{\left(2 r_{2}\right)^{2}}
$$

where $E_{04}$ is the electron interaction energy; $R$ is the size of a rhomb edge; $r_{1}$ and $r_{2}$ are the distances of oppositely located electrons from the nucleus which are related to the rhomb edge as follows: $r_{1}=R \sin \alpha$ and $r_{2}=$ $R \cos \alpha$; and $\alpha$ is the angle between an edge and the major diagonal.

Oxygen atom:

$$
\begin{aligned}
& E_{08}=-\frac{48 e^{2}}{r_{1}}-\frac{16 e^{2}}{r_{2}}+\frac{6 \Gamma}{r_{1}^{2}}+\frac{2 \Gamma}{r_{2}^{2}}+\frac{6 e^{2}}{2 r_{1} \sin \left(\frac{\pi}{6}\right)}+\frac{6 e^{2}}{2 r_{1} \sin \left(\frac{\pi}{3}\right)}+\frac{3 e^{2}}{2 r_{1}}+ \\
& +\frac{12 e^{2}}{R}+\frac{e^{2}}{2 r_{2}}+\frac{12 A}{R^{2}}+\frac{6 A}{\left(2 r_{1} \sin \left(\frac{\pi}{6}\right)\right)^{2}}+\frac{6 A}{\left(2 r_{1} \sin \left(\frac{\pi}{3}\right)\right)^{2}}+\frac{3 A}{\left(2 r_{1}\right)^{2}}+\frac{A}{\left(2 r_{2}\right)^{2}}
\end{aligned}
$$

where $E_{08}$ is the electron interaction energy in an oxygen atom; $r_{1}$ is the distance of electrons located at vertices 
of the pyramid base from the nucleus; $r_{2}$ is the distance of electrons located at vertices of the pyramids from the nucleus; $R$ is the length of a lateral edge of the pyramid. The values of $R, r_{1}$ and $r_{2}$ are connected by the relations: $r_{1}=R \sin \alpha$ and $r_{2}=R \cos \alpha$, where $\alpha$ is the angle between a lateral edge and the pyramid base.

The results of calculations of the binding energies of electrons in each of the proposed models of atoms are given in Table 1.

Table 1. Comparison of the theoretical and experimental values of binding energies of electrons in atoms.

\begin{tabular}{lllllllllll}
\hline Elements & $\mathrm{H}$ & $\mathrm{He}$ & $\mathrm{Li}$ & $\mathrm{Be}$ & $\mathrm{B}$ & $\mathrm{C}$ & $\mathrm{N}$ & $\mathrm{O}$ & $\mathrm{F}$ & $\mathrm{Ne}$ \\
\hline Exp & 13.595 & 78.969 & 203.44 & 399.03 & 671.08 & 1030.03 & 1485.74 & 2043.20 & 2713.03 & 3507.71 \\
$\mathrm{eV} \quad$ theor & 13.607 & 78.344 & 204.07 & 401.77 & 673.57 & 1029.40 & 1488.86 & 2037.00 & 2674.32 & 3504.59 \\
Error, \% & +0.1 & -0.8 & +0.3 & -0.3 & +0.4 & -0.1 & +0.2 & -0.3 & -1.4 & +0.1 \\
\hline
\end{tabular}

Thus, the possibility to construct the static models of atoms by the same principle with the same constants testifies, in our opinion, to the reality of the binomial potential.

\section{A Model for the Study of Near-Atom Regions}

Models for the study of the interaction of electrically neutral atoms with free charges were constructed on the basis of the models proposed by us (Gudym \& Andreeva, 2008), for neutral atoms (Figure 4). For example, if we imagine that the hydrogen atom consists of a proton and an electron located at the distance $r_{0}$ from it, then the model of the interaction for such a system with a free electron $e_{0}$ or a proton $p_{0}$ can be presented in the form shown in Figure 5.

The electron interaction energy $E$ is as follows:

$$
E=-\frac{e^{2}}{r_{0}}+\frac{\Gamma}{r_{0}^{2}}-\frac{e^{2}}{R}+\frac{\Gamma}{R^{2}}+\frac{e^{2}}{r}+\frac{A}{r^{2}} .
$$

where $A$ is the constant of the magnetic interaction of two electrons (Gudym \& Andreeva, 2008); $R$ is the distance between the electron $e_{0}$ or the proton $p_{0}$ and the atomic nucleus; $r_{0}$ is the equilibrium radius of a hydrogen atom;

$$
r=\sqrt{r_{0}^{2}+R^{2}-2 r_{0} R \cos \alpha}
$$

and $\alpha$ is the angle between the fixed coordinate axis and the direction $R$ to the free electron $e_{0}$ or the proton $p_{0}$.

The interaction energy $E$ of the proton of the hydrogen atom is given by the formula

$$
E=-\frac{e^{2}}{r_{0}}+\frac{\Gamma}{r_{0}^{2}}+\frac{e^{2}}{R}-\frac{\Gamma}{R^{2}}-\frac{e^{2}}{r}+\frac{\Gamma}{r^{2}}
$$

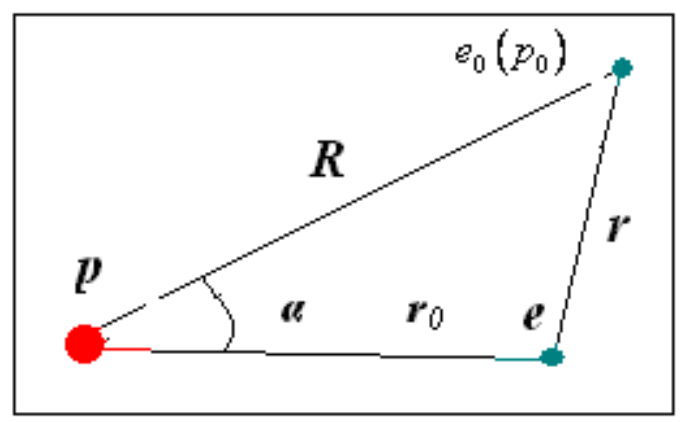

Figure 5. Model of interaction of a neutral hydrogen atom with trial charges: $\mathrm{e}_{0}$-electron and $\mathrm{p}_{0}$-proton 


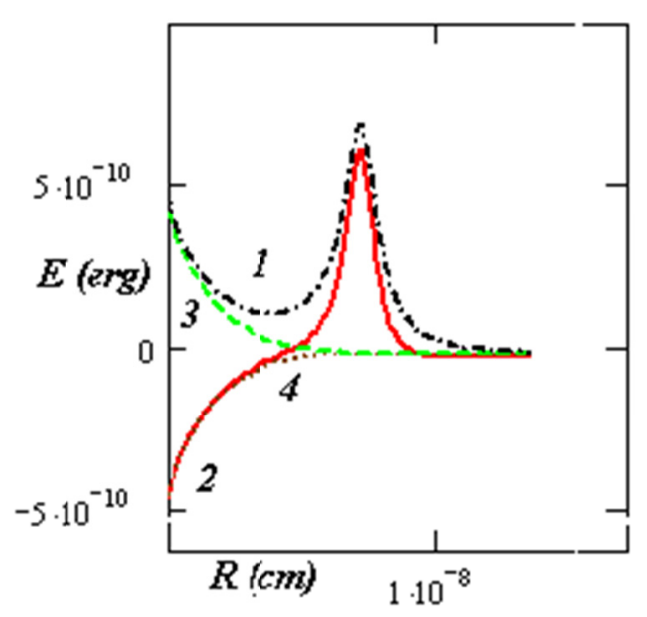

Figure 6. Interaction energy of a neutral hydrogen atom with trial charges (1, 3-electron and 2, 4-proton) the distance between them for angles: $1,2-9^{\circ}, 3,4-180^{\circ}$

Thus, by using Formulas (22) and (22') and setting values of $R$ and $\alpha$, we have the possibility to calculate the total energy of the "hydrogen atom - free charge" system for every point, where the extraneous electron $e_{0}$ or proton $p_{0}$ is located in the near-atom region. The sign and the modulus of the calculated energy will indicate the character of the interaction of the atom with a free charge. If the sign is positive, then the interaction is repulsive; for the negative sign, the interaction is attractive. In Figure 6, we demonstrate how the energy of the "neutral atom - free charge" system varies as a function of the distance of the free charge from the nucleus. It is clear that, by starting from infinity and to distances of approximately $10^{-8} \mathrm{~cm}$, only the attractive forces act between a neutral hydrogen atom and a free charge independent of the sign of the free charge for all directions.

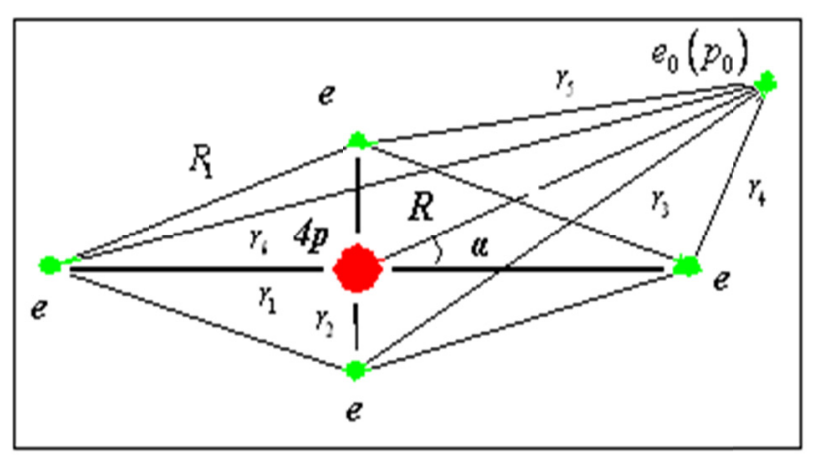

Figure 7. Model of interaction of a neutral beryllium atom withtrial charges: $\mathrm{e}_{0}$-electron and $\mathrm{p}_{0}$-proton

As another example of the model of interaction of an electrically neutral atom with a free charge, we took a beryllium atom (Figure 7). Within this model, we write analogously the interaction energy of an electron with a atom beryllium in the form

$$
\begin{aligned}
& E=\left[-\frac{8 e^{2}}{r_{1}}+\frac{2 \Gamma}{r_{1}^{2}}-\frac{8 e^{2}}{r_{2}}+\frac{2 \Gamma}{r_{2}^{2}}+\frac{4 e^{2}}{R_{1}}+\frac{e^{2}}{2 r_{1}}+\frac{e^{2}}{2 r_{2}}+\frac{4 A}{R_{1}^{2}}+\frac{A}{\left(2 r_{1}\right)^{2}}+\frac{A}{\left(2 r_{2}\right)^{2}}\right]+ \\
& +\left[-\frac{4 e^{2}}{R}+\frac{\Gamma}{R^{2}}+e^{2}\left(\frac{1}{r_{3}}+\frac{1}{r_{4}}+\frac{1}{r_{5}}+\frac{1}{r_{6}}\right)+A\left(\frac{1}{r_{3}^{2}}+\frac{1}{r_{4}^{2}}+\frac{1}{r_{5}^{2}}+\frac{1}{r_{6}^{2}}\right)\right]
\end{aligned}
$$

where 


$$
\begin{aligned}
& r_{3}=\sqrt{r_{1}^{2}+R^{2}-2 r_{1} \cdot R \cdot \cos \left(\frac{\pi}{2}+\alpha\right)}, r_{4}=\sqrt{r_{2}^{2}+R^{2}-2 r_{2} \cdot R \cdot \cos (\alpha)}, \\
& r_{5}=\sqrt{r_{1}^{2}+R^{2}-2 r_{1} \cdot R \cdot \cos \left(\frac{\pi}{2}-\alpha\right)}, r_{6}=\sqrt{r_{2}^{2}+R^{2}-2 r_{2} \cdot R \cdot \cos (\pi-\alpha)} .
\end{aligned}
$$

Here, $R$ is the distance from the atom center to the free charge, $\alpha$ is the angle between the direction from the atom center to the free charge and a fixed coordinate axis with the origin at the nucleus (Gudym \& Andreeva, 2007). Values of $R_{1}, r_{1}, r_{2}$ were taken from the calculation of the neutral atom.

For the interaction energy of a proton with a beryllium atom, we can write the formula

$$
\begin{aligned}
E & =\left[-\frac{8 e^{2}}{r_{1}}+\frac{2 \Gamma}{r_{1}^{2}}-\frac{8 e^{2}}{r_{2}}+\frac{2 \Gamma}{r_{2}^{2}}+\frac{4 e^{2}}{R_{1}}+\frac{e^{2}}{2 r_{1}}+\frac{e^{2}}{2 r_{2}}+\frac{4 A}{R_{1}^{2}}+\frac{A}{\left(2 r_{1}\right)^{2}}+\frac{A}{\left(2 r_{2}\right)^{2}}\right]+ \\
& +\left[\frac{4 e^{2}}{R}-\frac{\Gamma}{R^{2}}-e^{2}\left(\frac{1}{r_{3}}+\frac{1}{r_{4}}+\frac{1}{r_{5}}+\frac{1}{r_{6}}\right)+\Gamma\left(\frac{1}{r_{3}^{2}}+\frac{1}{r_{4}^{2}}+\frac{1}{r_{5}^{2}}+\frac{1}{r_{6}^{2}}\right)\right]
\end{aligned}
$$

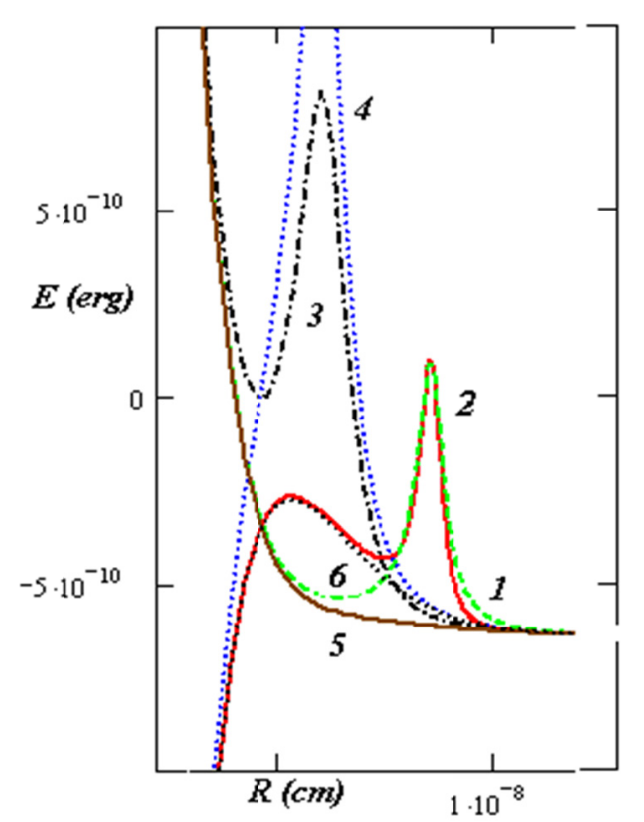

Figure 8. Interaction energy of a neutral beryllium atom with trial charges $(1,3,5$-electrons, 2, 4, 6-protons) the distance between them. In the plane of the atom at angles: $1,2-9^{\circ} ; 3,4-108^{\circ} ; 5$ and 6 --perpendicularly to the plane of the atom

In Figure 8, we present the variation of the interaction energy of free electric charges with a beryllium atom as a function of the distance between the free charge and the atom center for different directions to the free charge. Curves 1 and 2 give the interaction energy of a neutral atom of beryllium with electric charges as a function of the distance from the atom center for the angle $\alpha=9^{\circ}$. It is obvious that, at distances of the electric charge from the atom center more than $1 \cdot 10^{-8} \mathrm{~cm}$, the total energy of the system is negative. Hence, in this region, the neutral atom and electric charges irrespective of their charge are attracted. An analogous pattern is observed for all other directions to extraneous electric charges $(3,4,5,6)$. Thus, the example with a beryllium atom also indicates that, by starting from infinity and to distances of $\sim 1 \times 10^{-8} \mathrm{~cm}$, the electric charges with any sign undergo only the mutual attraction with neutral atoms.

An analogous attraction of free charges and neutral atoms is observed for all ten first atoms of the Mendeleev Periodic table.

It is obvious from Figures 6 and 8 that the character of the interaction of free charges with neutral atoms at 
intra-atomic distances is significantly more complicated than that observed at distances larger than $1 \times 10^{-8} \mathrm{~cm}$. But the consideration of this problem is outside of the present work.

\section{Conclusion}

The purpose of the present work was to clarify the nature of the gravitational interaction. To this end, we developed a method for the calculation of a numerical value of the interaction energy of the atomic system with trial charges located at various points of the near-atom region. Then we considered the near-atom regions for ten first elements of the Mendeleev Periodic table. On this way, the analysis of electrostatic fields present in the near-atom regions for electrically neutral atoms allows us to understand the reason for the appearance of the attraction between electrically neutral bodies.

For the complete comprehension of the mechanism of appearance of the gravitational interaction, we need to consider this difference not only near a nucleus or at the boundary of an atom, but also at large distances from the atom.

The difference between the Coulomb and binomial potentials near a nucleus results in the dominance of the repulsive component of the binomial potential at small distances as compared with the attractive Coulomb component. This makes an atom to be a stable system.

Here, we should like to make a remark in order that the reader will not identify the stability of an orbit and the stability of an atom. When we consider the former in the sense of the Bertrand theorem, we mean only a one-electron system of the hydrogen-atom type. But if we write generally about the stability of an atom, we mean many-electron systems. According to our static model (Figure 4), electrons and the nucleus of such many-electron system are in the stable equilibrium position with respect to one another. In other words, each electron is located in the own potential well, where there is no orbital motion, as distinct from the other old models of atoms. Hence, any connection between the stability of orbits and the stability of an atom has no physical sense.

Let us now consider (qualitatively) how the ratio of attractive and repulsive forces will be changed, as the distance from a nucleus will increase. In this case, the repulsive force decreases faster than the attractive Coulomb one. Therefore, they become equal in modulus at some distance from the nucleus. This distance gives the size of atom. For greater distances from the atom, the repulsive force will be less in modulus that the attractive Coulomb one. It is essential that the repulsive force does not disappear completely. As the distance from the atom increases, both forces decrease, and the dominance of the Coulomb component will increase more and more. Thus, the residual electric field exists outside the limits of each electrically neutral atom. The interaction of such fields is responsible for the attraction between electrically neutral atoms, molecules, and, generally, macrobodies. All this is illustrated in Figures 1, 6, and 8.

At the present time, many theories of gravitation are available. But their common shortcoming is that they cannot join the gravity with any other interaction known in physics. But we succeeded, for the first time, to find such general criterion that allows us to connect the gravity with the electrostatic interaction. At this point, our theory made a significant step forward on the way of cognition of the nature of gravitation as compared with the other available theories related to the gravity.

Thus, we have succeeded to strictly prove that the gravitational and electrostatic interactions have the same nature, so that the gravitational interaction is not caused by some unique field created by electrically neutral bodies. In our opinion, the gravity is related to the well-known electrostatic field and possesses, like all electric fields, the properties of Faraday-Maxwell force fields. We note that our conclusion agrees completely with the results of the Logunov's relativistic theory of gravitation (Logunov, 1987).

Hence, the attractive forces existing between electrically neutral physical bodies (gravitational forces) arise as a result of the electrostatic interaction of electrically neutral atoms with the electric charges of other, also electrically neutral, atoms.

The equivalence of the Coulomb and Newton laws can be also shown by comparing the dimensions of the quantities in the formulas of these laws. Let us consider the equalities

$$
E=\frac{e^{2}}{r}=\gamma \frac{M^{2}}{r}=\frac{L^{3} \cdot M}{T^{2} \cdot L}=\frac{L^{3} \cdot M^{2}}{M \cdot T^{2} \cdot L}=\frac{L^{2} \cdot M}{T^{2}}=\operatorname{erg}
$$

Where $\gamma$ is the gravitational constant; $M$ is the mass; $L$ is the length; and $T$ is the time.

In (25), the second and third equalities present, respectively, the Coulomb and Newton laws. The substitution of 
the corresponding dimensions (fourth and fifth equalities) leads us to the same energy unit in the CGSE system, which demonstrates once more the equivalence of the Coulomb and Newton laws.

This connection follows easily from the following reasoning.

As is known, all electrically neutral bodies in the Nature reveal always the mutual attraction. In our work, we have shown that all neutral atoms and molecules (see Figures 5-8) manifest also the mutual attraction to the remote electric charges irrespective of whether these charges are positive or negative, i.e., to electrons and nuclei on the same basis.

In other words, we have shown that the attractive forces between electrically neutral bodies bear the electrostatic character. Earlier, the attractive forces between bodies were called the gravity forces. Hence, we can surely assert that both electrostatic and gravitational forces have the same nature, i.e., they are equivalent.

Generally speaking, the notion "gravitational interaction" establishes only the fact of attraction of bodies. Since we have found that the attraction between bodies appears as a result of the electrostatic interaction, we have the firm basis to identify the gravity with the electrostatic interaction.

\section{References}

Born, M. (1946). Atomic Physics. New York: Hafner.

Gudym, V. K., \& Andreeva, E. V. (2007a). Motion of an electron in the field of a binomial potential of a proton. The Old and New Concepts of Physics, 4(4), 553-566. http://dx.doi.org/10.2478/v10005-007-0025-8

Gudym, V. K., \& Andreeva, E. V. (2007b). Binomial potential of the electron-proton interaction as an alternative to the Coulomb law. Journal of Surface Investigation, 1(2), 223-228. http://dx.doi.org/10.1134/S1027451007020218

Gudym, V. K., \& Andreeva, E. V. (2007c). Poverkhn. 4, 1.

Gudym, V. K., \& Andreeva, E. V. (2008a). The energy of the binding of multielectronic structures in the field of binomial potential. The Old and New Concepts of Physics, 5(3), 435-458. http://dx.doi.org/10.2478/v10005-007-0040-9

Gudym, V. K., \& Andreeva, E. V. (2008b). Poverkhn. 10, 86.

Gudym, V. K., \& Andreeva, E. V. (2013). Quantum mechanics of a hydrogen atom: A new look at problems and a new approach to their explanation. Physics Essays, 26(2), 263-281. http://dx.doi.org/10.4006/0836-1398-26.2.263

Hofstadter, R. (1956). Electron scattering and nuclear structure. Rev. Mod. Phys., 28, 214-254. http://dx.doi.org/10.1103/RevModPhys.28.214

Levich, V. G. (1969). Course of Theoretical Physics. Vol. 1, Moscow: Fizmatgiz, p. 122.

Logunov, A. A. (1987). Lectures on relativity theory and gravitation: Modern analysis of the problem. Moscow: Nauka.

Mead, C. (2001). American Spectator, Sep/Oct 34 Issue 7, 68.

Zhirnov, N. I. (1980). Classical Mechanics, Prosveschenie, Moscow, 1980 (in Russian).

\section{Appendix A.}

The solution of system of the Equations (2)

$$
-\frac{e^{2}}{r_{0}}+\frac{\Gamma}{r_{0}^{x}}=E_{0}, \quad \frac{e^{2}}{r_{0}^{2}}-\frac{x \cdot \Gamma}{r_{0}^{x+1}}=0
$$

By reducing this system to the form

$$
-\frac{e^{2}}{r_{0}}+\frac{\Gamma}{r_{0}^{x}}=E_{0}, \quad \frac{e^{2}}{x r_{0}}-\frac{\Gamma}{r_{0}^{x}}=0
$$

And by adding term-by-term, we obtain the equality

$$
-\frac{e^{2}}{r_{0}}+\frac{e^{2}}{x r_{0}}=E_{0} .
$$

It can be easily transformed to the relation 


$$
\frac{1}{x}-1=\frac{E_{0} \cdot r_{0}}{e^{2}}
$$

By substituting the numerical values of $E_{0}, r_{0}$, and $e^{2}$ from handbooks, we obtain that $x=2$ with the error in the fifth decimal point (1.999934). This corresponds to the accuracy of the relevant constants

$$
E_{0}=-2.17977 \times 10^{-11}, e=4.80298 \times 10^{-10}, r_{0}=0.52917 \times 10^{-8} .
$$

With this value of $x$, the second equation of system (2) becomes

$$
\Gamma=\frac{e^{2} \cdot r_{0}}{2}
$$

Thus, the above-presented solution of system (2) for $\Gamma$ and $x$ gives

$$
\Gamma=6.10276 \cdot 10^{-28} \text { (CGSE units) and } x=2
$$

We note that the special attention should be given to the value of $x$ equal to 2 . This is of importance because this value was obtained as a result of the exact solution of the system of two equations corresponding to the ground state of a hydrogen atom. We emphasize that only such value of $x$ for the binomial potential allows us to exactly integrate the equations of motion. In other words, this result makes the binomial potential to be applicable to other problems of atomic physics.

\section{Appendix B.}

The solution of the Equation (4)

$$
E=\frac{m \dot{r}^{2}}{2}+\frac{M^{2}}{2 m r^{2}}-\frac{e^{2}}{r}+\frac{\Gamma}{r^{2}}
$$

In the general case, this equation has the form

$$
E=\frac{m \dot{r}^{2}}{2}+\frac{M^{2}}{2 m r^{2}}+U(r)
$$

This yields

$$
\dot{r}=\frac{d r}{d t}=\sqrt{\frac{2}{m}[E-U(r)]-\frac{M^{2}}{m^{2} r^{2}}} \quad t=\int \frac{d r}{\sqrt{\frac{2}{m}[E-U(r)]-\frac{M^{2}}{m^{2} r^{2}}}}
$$

Using the well-known conservation law of the angular moment

$$
M=m r^{2} \dot{\varphi},
$$

We write

$$
\frac{d \varphi}{d t}=\frac{M}{m r^{2}} \text { or } d \varphi=\frac{M}{m r^{2}} d t
$$

Substituting $d t$ to the last relation, we obtain

$$
\varphi=\int \frac{\frac{M}{r^{2}} d r}{\sqrt{2 m[E-U(r)]-\frac{M^{2}}{r^{2}}}} .
$$

This formula presents the general solution of the posed problem to determine the connection between the radius-vector and the angle of its rotation. That is, we obtained the equation for trajectories.

Substituting the binomial potential $U(r)=-\frac{e^{2}}{r}+\frac{\Gamma}{r^{2}}$ to the last formula, we arrive at the expression 


$$
\varphi=\int \frac{\frac{M}{r^{2}} d r}{\sqrt{2 m\left[E+\frac{e^{2}}{r}-\frac{\Gamma}{r^{2}}\right]-\frac{M^{2}}{r^{2}}}}
$$

The integration was performed with the use of the tabular formula

$$
\int \frac{d r}{r \sqrt{a+b r+c r^{2}}}=\frac{1}{\sqrt{-a}} \arcsin \frac{2 a+b r}{r \sqrt{b^{2}-4 a c}} .
$$

As a result, we have

$$
\varphi=\frac{M}{\sqrt{2 m \Gamma+M^{2}}} \arcsin \frac{m e^{2} r-\left(2 m \Gamma+M^{2}\right)}{r \sqrt{m^{2} e^{4}+2 m E\left(2 m \Gamma+M^{2}\right)}} .
$$

Introducing the designations

$$
\varepsilon=\sqrt{1+\frac{2 E\left(2 m \Gamma+M^{2}\right)}{m e^{4}}}, P=\frac{2 m \Gamma+M^{2}}{m e^{2}}, \text { and } k=\frac{\sqrt{2 m \Gamma+M^{2}}}{M},
$$

We obtain the formula for a motion trajectory in the form

$$
r=\frac{P}{1-\varepsilon \sin (k \phi)} .
$$

Generally speaking, the reader can have some doubts as to the reality of the equation of orbits (5). At the present time, it is commonly accepted that this problem is solved in a finite form and describes the closed trajectories only with the fields proportional to $\frac{1}{r}$ and $\frac{k r}{2}$, according to the Bertrand theorem. But this is not the case, since an important thing is omitted. The Bertrand theorem is valid only if we consider the closed trajectories under arbitrary initial conditions, i.e., under any initial values of the energy and the angular momentum (Zhirnov, 1980). Under definite initial conditions, the closed trajectories can be obtained with other potentials. Our exact solution of this problem with the binomial potential indicates that if the Coulomb law is supplemented by a positive term proportional to $\frac{1}{r^{2}}$, then the two-body problem can be also solved in a finite form. But it should be mentioned that, in order that the trajectories be closed in our case, we must set the corresponding initial conditions, i.e., a certain collection of values of the energy and the angular momentum. In Appendix 1, we gave attention to the value of the exponent. If $x \neq 2$, we cannot obtain the exact solution of Equation (4). Thus, we have found one more potential, for which the two-body problem admits a finite solution.

\section{Copyrights}

Copyright for this article is retained by the author(s), with first publication rights granted to the journal.

This is an open-access article distributed under the terms and conditions of the Creative Commons Attribution license (http://creativecommons.org/licenses/by/3.0/). 